\title{
COFINAL FAMILIES OF COMPACTA IN SEPARABLE METRIC SPACES
}

\author{
FONS VAN ENGELEN
}

(Communicated by Dennis K. Burke)

\begin{abstract}
We show that if $X$ is a $\Pi_{1}^{1}$-set, then the family of compact subsets of $X$ contains a cofinal (w.r.t. inclusion) subset of cardinality $\mathbf{d}$; the same is true if $X$ is $\Pi_{3}^{1}$, under strong set-theoretic hypotheses.
\end{abstract}

All spaces are separable and metrizable. For all undefined notions and unproved assertions, see Engelking [3], Kuratowski [4], and Moschovakis [6]. We work in ZFC, throughout. We assume familiarity with van Douwen's handbook article [1]; for this note, however, it will be convenient to let $\mathscr{K}(X)$ be the space of all nonempty compact subsets of $X$ with the Vietoris topology, rather than the set of all compacta in $X$. Van Douwen asks whether $\operatorname{cof}(\mathscr{K}(X))=k(X)=\mathbf{d}$ if $X$ is analytic (i.e. $\Sigma_{1}^{1}$ ), or at least absolutely Borel (i.e. $\left.\Delta_{1}^{1}\right)$, and presumably non- $\sigma$-compact, having shown (among other things) that always $k c(X) \leq k(X) \leq \operatorname{cof}(\mathscr{K}(X))$, that $k c(X)=\mathbf{d}$ if $X$ is a non- $\sigma$-compact and analytic, and that $k c(X)=k(X)=\operatorname{cof}(\mathscr{K}(X))=\mathbf{d}$ if $X$ is a non- $\sigma$-compact absolute $F_{\sigma \delta}$. Our main result is that $\operatorname{cof}(\mathscr{K}(X)) \leq \mathbf{d}$ if $X$ is coanalytic (i.e. $\Pi_{1}^{1}$ ); thus, for non- $\sigma$-compact absolute Borel sets $X, k c(X)=$ $k(X)=\operatorname{cof}(\mathscr{K}(X))=\mathbf{d}$. Extensions to sets of higher complexity are also discussed.

Proposition. For any space $X, \operatorname{cof}(\mathscr{K}(X))=k c(\mathscr{K}(X))$.

ProOF. Let $\mathscr{L}$ be cofinal in $\mathscr{K}(X)$. Then each element of $\mathscr{K}(X)$ is contained in some $L \in \mathscr{L}$; so $\mathscr{K}(X)=\bigcup_{L \in \mathscr{L}} \mathscr{K}(L)$, where $\mathscr{K}(L)$ is compact. So $k c(\mathscr{K}(X)) \leq$ $\operatorname{cof}(\mathscr{K}(X))$.

Conversely, let $\mathscr{C}$ be a covering of $\mathscr{K}(X)$ by compact sets. Then for each $C \in \mathscr{C}$, $\bigcup C$ is compact. Let $\mathscr{L}=\{\bigcup C: C \in \mathscr{C}\}$; we claim that $\mathscr{L}$ is cofinal in $\mathscr{K}(X)$. Indeed, if $\varnothing \neq K \subseteq X$ is compact, then $K \in C$ for some $C \in \mathscr{C}$; hence, $K \subseteq \cup C$, and we are done.

At this point, let us remark that by van Douwen [1, Lemma 8.9], for all computations of $k c, k$ and cof, we can restrict ourselves to subsets of the Cantor set $2^{\omega}$, since every $\Pi_{n}^{1}, \Sigma_{n}^{1}, \Delta_{n}^{1}$ subset of the Hilbert cube is the perfect image of a subset of $2^{\omega}$ of the same class.

LEMMA. Let $X$ be a subset of the Cantor set $2^{\omega}$. If $n \in \mathbf{N}$, and $X$ is $\Pi_{n}^{1}$, then so is $\mathscr{K}(X) \subseteq \mathscr{K}\left(2^{\omega}\right) \approx 2^{\omega}$.

PROOF. $\{(x, K): x \in K\}$ is closed in $2^{\omega} \times \mathscr{K}\left(2^{\omega}\right)$. So $\{(x, K): x \in K\} \cap\left(2^{\omega} \backslash X\right) \times$ $\mathscr{K}\left(2^{\omega}\right)$ is $\Sigma_{n}^{1}$, hence the projection $\left\{K \in \mathscr{K}\left(2^{\omega}\right)\right.$ : for some $\left.x \in 2^{\omega} \backslash X, x \in K\right\}$ of

Received by the editors October 12, 1987.

1980 Mathematics Subject Classification (1985 Revision). Primary 54H05.

Research partially supported by the Netherlands organisation for the advancement of pure research (Z.W.O.) and the University of California at Berkeley. 
this set onto $\mathscr{K}\left(2^{\omega}\right)$ is also $\Sigma_{n}^{1}$. But the complement of the projected set is just $\left\{K \in \mathscr{K}(X): K \cap\left(2^{\omega} \backslash X\right)=\varnothing\right\}=\mathscr{K}(X)$, so $\mathscr{K}(X)$ is $\Pi_{n}^{1}$.

THEOREM. Let $X$ be any $\Pi_{1}^{1}$-set. Then $\operatorname{cof}(\mathscr{K}(X)) \leq \mathrm{d}$.

ProOF. By the above remark, let $X \subseteq 2^{\omega}$. By the lemma, $\mathscr{K}(X)$ is $\Pi_{1}^{1}$, and by the proposition $\operatorname{cof}(\mathscr{K}(X))=k c(\mathscr{K}(X))$. By Luzin-Sierpiński [5], a $\Pi_{1}^{1}$-set is the union of $\aleph_{1}$ Borel sets, $\mathscr{K}(X)=\bigcup_{\alpha<\omega_{1}} B_{\alpha}$. Now since $B_{\alpha}$ is a continuous image of $\omega^{\omega}$, we have $k c(X) \leq k c\left(\omega^{\omega}\right)=\mathbf{d}$; thus $k c(\mathscr{K}(X)) \leq \mathbf{d} . \aleph_{1}=\mathbf{d}$.

COROLLARY. (a) Let $X$ be a non- $\sigma$-compact absolute Borel set; then $k c(X)=$ $k(X)=\operatorname{cof}(\mathscr{K}(X))=\mathbf{d}$.

(b) Let $X$ be nonlocally compact and coanalytic; then $\operatorname{cof}(\mathscr{K}(X))=\mathbf{d}$.

PROOF. (a) follows from the theorem and van Douwen [1, Theorem 8.10(e)]; (b) follows from the theorem and van Douwen [1, Fact 8.1(c), Lemmas 8.3 and 8.4].

The proof of the theorem shows in fact that $\operatorname{cof}(\mathscr{K}(X)) \leq \mathbf{d}$. $\kappa$ if $\mathscr{K}(X)$ can be written as a union of $\kappa$ Borel sets. If $X$ is $\Pi_{2}^{1}$, then so is $\mathscr{K}(X)$ by the lemma, and it is a theorem of Martin (see Moschovakis [5]) that if $\Sigma_{1}^{1}$ games are determined (and AC holds) then any $\Sigma_{3}^{1}$-set is a union of $\aleph_{2}$ Borell sets; if $X$ is $\Pi_{3}^{1}$, then so is $\mathscr{K}(X)$, and the same theorem of Martin says that if $\Delta_{2}^{1}$-games are determined (and AC holds) then any $\Sigma_{4}^{1}$-set is a union of $\aleph_{3}$ Borel sets. Furthermore, from a theorem of Steel [7] it can easily be deduced (compare van Engelen [2, Lemma 4.5.5]) that, if $\Lambda$ is some $\Sigma_{n}^{1}, \Pi_{n}^{1}$, or $\Delta_{n}^{1}$, then determinancy of $\Lambda$ games implies that every non- $\sigma$-compact set in $\Lambda$ contains a closed copy of $\omega^{\omega}$. Combining these remarks with van Douwen's results, we have

COROLLARY. (a) Let $X$ be analytic and non- $\sigma$-compact. If analytic games are determined, and $\mathbf{d} \geq \aleph_{2}$, then $k c(X)=k(X)=\operatorname{cof}(\mathscr{K}(X))=\mathbf{d}$.

(b) Let $X$ be $\Pi_{2}^{1}$. If $\Sigma_{1}^{1}$-games are determined, and $\mathbf{d} \geq \aleph_{2}$, then $\operatorname{cof}(\mathscr{K}(X)) \leq \mathbf{d}$; if furthermore $X$ is non- $\sigma$-compact and $\Pi_{2}^{1}$-games are determined, then $k c(X)=$ $k(X)=\operatorname{cof}(\mathscr{K}(X))=\mathbf{d}$.

(c) Let $X$ be $\Pi_{3}^{1}$. If $\Delta_{2}^{1}$-games are determined and $\mathbf{d} \geq \aleph_{3}$, then $\operatorname{cof}(\mathscr{K}(X)) \leq \mathbf{d}$; if furthermore $X$ is non- $\sigma$-compact, and $\Pi_{3}^{1}$-games are determined, then $k c(X)=$ $k(X)=\operatorname{cof}(\mathscr{K}(X))=\mathbf{d}$.

Recent results of Jackson combined with well-known results of descriptive set theory imply that if determinancy holds for $L(\mathscr{R})$ (and AC holds) then for each $n$ we can find a natural number $k(n)$ such that each $\Pi_{n}^{1}$-set is a union of $\aleph_{k(n)}$ Borel sets. Thus

COROLlaRY. If $\operatorname{Det}(L(\mathscr{R}))$ and $\mathbf{d} \geq \aleph_{k(n)}$, then for $X$ a non- $\sigma$-compuct $\Pi_{n}^{1}$ set, $k c(X)=k(X)=\operatorname{cof}(\mathscr{K}(X))=\mathbf{d}$.

\section{REFERENCES}

1. E. K. van Douwen, The integers and topology, Handbook of Set Theoretic Topology, NorthHolland, Amsterdam, 1984, pp. 111-167.

2. F. van Engelen, Homogeneous zero-dimensional absolute Borel sets, CWI Tract 27, 1986.

3. R. Engelking, General topology, PWN, Warszawa, 1977.

4. K. Kuratowski, Topology I, Academic Press, New York, 1966.

5. N. Luzin and W. Sierpiński, Sur quelques propriétés des ensembles (A), Bull. Int. Acad. Sci. Cracovie Série A Sci. Math, (1918), 35-48. 
6. Y. Moschovakis, Descriptive set theory, North-Holland, Amsterdam, 1980.

7. J. Steel, Analytic sets and Borel isomorphisms, Fund. Math. 108 (1980), 83-88.

Faculteit Wiskunde \& Informatica, Der VRiJe Universiteit, De BoelelaAN 1081, 1081 HV AMSTERDAM, THE NETHERLANDS

Current address: Faculteit Wiskunde \& Informatica, Uinversiteit van Amsterdam, Roetersstraat 15, 1018 WB Amsterdam, The Netherlands 